\title{
Research on an Improved Wireless Sensor Networks Effective Coverage Method
}

\author{
Wenli Lei ${ }^{1,2 *}$ and Fubao Wang ${ }^{1}$ \\ ${ }^{1}$ School of Electronics and Information, Northwestern Polytechnical University, Xi'an, \\ 710072 China \\ ${ }^{2}$ School of Physics and Electronic Information, Yan'an University, Yan'an 716000 , \\ China \\ lei.wenli@163.com
}

\begin{abstract}
An improved wireless sensor networks effective coverage method based on differential evolution algorithm is proposed in this paper, which using quadratic interpolation method to improve the algorithm performance to further optimize the effective coverage of wireless sensor network nodes. Simulation results show that, compared to DE algorithm, the new algorithm can improve local search capabilities, reduce the computational cost of the algorithm, accelerate standards DE convergence, improve the accuracy of solution, and has some reference value for optimization application of wireless sensor network.
\end{abstract}

Keywords: differential evolution; wireless sensor networks coverage; algorithm

\section{Introduction}

With the progress of MEMS, sensor technology and communications technology, wireless sensor network has become more and more closely linked with the modern industrial production and daily life. Wireless sensor network coverage is an important indicator to measure the normal operating range of the wireless sensor network in the monitoring area. Coverage can be divided into deterministic and random coverage in accordance with deployment [1]. Deterministic coverage refers to the WSN fixed or are completely known, the node configuration in advance. In this case, coverage issue transformed into a special geometry problem or path-planning problem. Random cover is for a lot of the actual environment, in most applications, we find it difficult to know the specifics of the actual monitoring environment, which requires sensor nodes can complete the coverage task of the monitored area without knowing the specific location of sensor nodes. According to the node, type coverage can be divided into two types of static coverage and dynamic coverage [2]. The static coverage means that nodes will not change its position in the network after being deployed, which can be divided into point or regional coverage, barrier coverage. Compared with static coverage, dynamic coverage refers to the enhanced node is capable to move in the network based on a certain optimization strategy, in order to change its coverage position. Dynamic coverage has a distinct advantage in energy efficiency, communication quality and channel capacity, data transmission accuracy, moving target tracking accuracy, network coverage and connectivity, and network survival cycle, thus it received increasing attention [3].

This paper presents an improved wireless sensor networks effective coverage method based on differential evolution algorithm, which proposed using quadratic interpolation method to improve the algorithm performance to further optimize the effective coverage of wireless sensor network nodes [4]. This method reduces the computational cost of the algorithm without increasing the other hardware devices, and improves the efficiency of 
the node deployment. Simulation results show that the proposed algorithm has better effect on node deployment.

\section{Problem Description}

Assume that monitoring regional $\mathrm{A}$ is the two-dimensional plane, which is divided into $m \times n$ grid point [5], collection of sensor nodes on the monitored area is expressed by formula (1).

$$
\mathrm{S}=\left\{\mathrm{s}_{1}, \mathrm{~s}_{2}, \cdots \mathrm{s}_{\mathrm{n}}\right\}
$$

Where, $\mathrm{S}_{\mathrm{i}}=\left\{\mathrm{s}_{1}, \mathrm{~s}_{2}, \mathrm{~s}_{\mathrm{n}}\right\}$ is the coverage model of node $\mathrm{i},\left(\mathrm{x}_{\mathrm{i}}, \mathrm{y}_{\mathrm{i}}\right)$ is the coordinate of the node $\mathrm{i}, r_{s}\left(s_{i}^{k}\right)$ is a perception radius of node $\mathrm{S}_{\mathrm{i}}, r_{c}\left(s_{i}^{k}\right)$ is a communication radius of node $\mathrm{S}_{\mathrm{i}}$, and $r_{c}\left(s_{i}^{k}\right) \geq 2 r_{s}\left(s_{i}^{k}\right)$, which ensure that nodes are deployed to form a connected network. GPS or some localization algorithms can accurately obtain all sensing nodes location.

For any grid point $\mathrm{P}_{\mathrm{j}}$, the Euclidean distance between sensor nodes $s_{i}^{k}$ and grid points $\mathrm{P}_{\mathrm{j}}$ is:

$$
d\left(s_{i}, \mathrm{p}_{j}\right)=\sqrt{\left(x_{i}-x_{j}\right)^{2}+\left(y_{i}-y_{j}\right)^{2}}
$$

Using binary function model of nodes, that is, when the $\mathrm{d}\left(s_{i}^{k}, \mathrm{P}_{\mathrm{j}}\right)$ is less than the node $s_{i}^{k}$ perception radius, then $\mathrm{P}_{\mathrm{j}}$ grid points has been covered by node $s_{i}^{k}$, that is,

$$
c_{b}\left(s_{i}^{k}, p_{j}\right)=\left\{\begin{array}{rr}
1 & \text { ifd }\left(s_{i}^{k}, p_{j}\right)<r_{s}\left(s_{i}^{k}\right) \\
0 & \text { otherwise }
\end{array}\right.
$$

When the distance between the grid points $(\mathrm{x}, \mathrm{y})$ to the sensor nodes $\mathrm{i}$ is not greater than the sensing range $\mathrm{r}$, that is considered the point $(\mathrm{x}, \mathrm{y})$ is covered by sensor nodes $\mathrm{i}$ and the complement of $\mathrm{I}_{\mathrm{i}}$ is $\bar{I}_{i}$, using equation (4) to represent:

$$
p\left\{\bar{I}_{i}\right\}=1-p\left\{I_{i}\right\}=1-p_{\text {cov }}\left(x, y, c_{i}\right)
$$

Expressing the event that point ( $x, y)$ is not covered by the i sensor nodes, if $I_{i}$ and $I_{j}$ is irrelevant, there is formulas (5) available:

$$
p\left\{I_{i} U I_{j}\right\}=1-p\left\{\bar{I}_{i} U \bar{I}_{j}\right\}=1-p\left\{\overline{I_{i}}\right\} \cdot p\left\{\bar{I}_{j}\right\}
$$

As long as the point $(\mathrm{x}, \mathrm{y})$ is covered by one node in the node set $\mathrm{C}$, that the grid point $(\mathrm{x}, \mathrm{y})$ is considered covered by the node set $\mathrm{C}$, so the probability of grid point $(\mathrm{x}, \mathrm{y})$ covered by a node set is the union of $I_{i}$, assuming all random events $I_{i}$ are independent of each other, then the coverage probability of the node set $\mathrm{C}$ can be calculated by the formula (6).

$$
p_{\text {cov }}(x, y, c)=p\left\{\bigcup_{i=1}^{N} I_{i}\right\}=1-p\left\{\bigcup_{i=1}^{N} \bar{I}_{i}\right\}=1-\prod_{i=1}^{N}\left(1-p_{\text {cov }}\left(x, y, c_{i}\right)\right)
$$

The formula (6) show that if the point $(\mathrm{x}, \mathrm{y})$ is not covered by all sensor nodes, then the point $(\mathrm{x}, \mathrm{y})$ is not covered point; otherwise, that consider the points $(\mathrm{x}, \mathrm{y})$ is covered by a node set.

Coverage rate of the network is defined as the ratio of the covered area by the node to the whole monitoring area, namely 


$$
\operatorname{Max} F_{i}=\frac{\sum_{j=1}^{N} c_{b}\left(p_{j}\right)}{N}
$$

And Formula (7) is used as the fitness function.

Wherein, $\mathrm{C}_{\mathrm{b}}\left(\mathrm{p}_{\mathrm{j}}\right)$ represents obtained coverage performance of all the sensor nodes in a grid point according to the formula(3).

The formula (7) shows that, the greater the value of the fitness function, the greater the coverage rate, the better the effect of the network coverage. By calculating the optimal solution of maximize fitness function, the optimized position estimation of the node can be obtained, and the coverage rate of the network can be improved.

\section{WSN Nodes Distribution Optimization Algorithm based on Differential Evolution}

\subsection{Differential Evolution Algorithm}

As a random, social and based on population of evolution algorithm, the concept of difference evolution algorithm initially proposed by Rainer Storn and Kenneth Price $\mathrm{Yu}$ in 1995 [6-7]. The algorithm is a bionic and intelligent method calculation algorithm which simulating natural evolution law of "Survival of the fittest". Difference evolution algorithm is a evolution algorithm, which has characteristics of strong global search capability, good robustness, simple general, convergence speed fast, and less control parameters. Differential evolution algorithm is an efficient global optimal solution search algorithm proved by the practice, which is widely used to solve complex global optimization problem, and has become an important tool in the field of computing science, information science and applied science, and gradually became one of the most important intelligent algorithms [8-9].

Differential evolution algorithm is inspired by the genetic mechanism of population evolution in nature, which is mainly composed of three basic elements: individual fitness evaluation, differential operation and parameter setting. (1) individual fitness evaluation. Differential evolution algorithms does not require additional external information in the process of evolution, and differential operation is realized and oriented mainly by the fitness function, according to the evaluation value of fitness function to evaluate pros and cons of the individual or solution. In general, the fitness evaluation function is defined according to the specific problem, which is used as the basis of differential evolution operation. (2) differential operation. The basic differential evolution algorithm and the genetic algorithm is similar, using three differential operations, respectively for the mutation operator, crossover operator, selection operator, only two evolutionary algorithm operators in a different order, differential evolution algorithm sequence of operations is mutation, crossover and selection [10-12]. DE algorithms process is shown in the following Figure 1. 


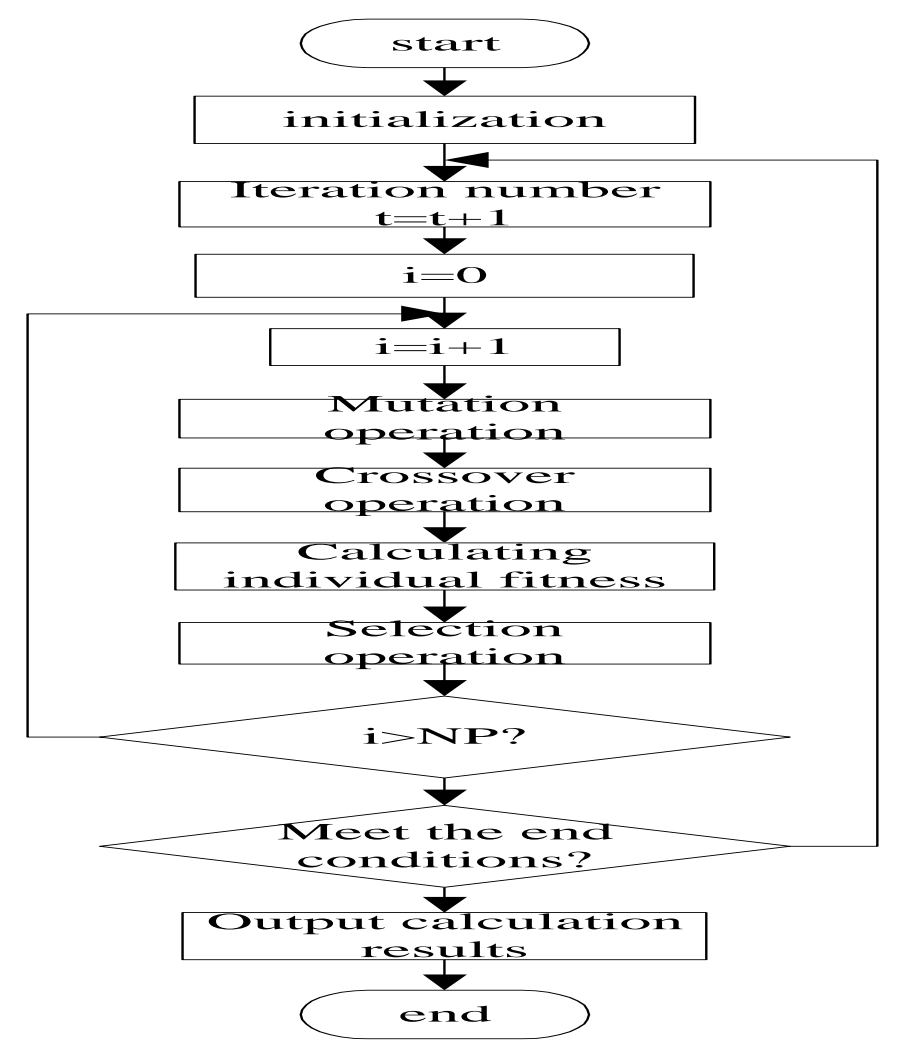

Figure 1. The Flow Chart of DE Algorithm

Let D-dimensional continuous space vector $\mathrm{X}=(\mathrm{X} 1, \mathrm{X} 2, \ldots, \mathrm{XD}) \in \mathrm{S}\left(S \subseteq R^{D}\right)$ is the decision vector of optimization problem $\mathrm{f}(\mathrm{x})$, and the optimization goal is to find the optimal $x \dagger$ to make the objective function(or fitness function) $f(x)$ maximum, that is, $\forall X \subseteq S$, there is $\mathrm{f}(\mathrm{x} \dagger) \geq \mathrm{f}(\mathrm{x})$. Procedure of $\mathrm{DE}$ algorithm is described as follows.

(1) The initial population. First determine the initial population of NP individuals. In the population, individual vector in the population is called the objective vector; the current generation of $i$-th objective vector is expressed as:

$$
X_{i, G}=\left(X_{1 i, G}, X_{2 i, G}, \cdots, X_{D i, G}\right)
$$

Where $\mathrm{G}=0,1, \ldots, \mathrm{Gmax}, \mathrm{G}=0$ is the initialization populations vector, $\mathrm{Gmax}$ is maximum number of generations. In the initialization process, we often want the objective vector be distributed as uniformly as possible in the solution space. Therefore, the i-th initialization objective vector can be written as:

$$
X_{j, i}=X_{j, \min }+\operatorname{rand}_{j, i}(0,1) *\left(X_{j, \max }-X_{j, \min }\right)
$$

Among them, the $\mathrm{j}$ is the $\mathrm{j}$-th parameter of the $\mathrm{i}$-th objective vector. $X_{\min }=\left\{X_{1, \min }, X_{2, \min }, \ldots X_{D, \min }\right\}$ and $X_{\max }=\left\{X_{1, \max }, X_{2, \max }, \ldots X_{D, \max }\right\}$ represent the boundary of objective vector.

(2) Mutation operator. After mutation operation, the DE algorithm generates the variation vector $\mathrm{V}_{\mathrm{iG}}$. In this paper, the "DE/rand/1" strategy is used to generate the variation vector $X_{i G}$ of each objective vector $V_{i G}$.

$$
V_{i, G}=X_{r 1, G}+F \cdot\left(X_{r 2, G}-X_{r 3, G}\right)
$$


Among them, the $\mathrm{r} 1, \mathrm{r} 2, \mathrm{r} 3$ are randomly selected positive integer from [1, NP], and $\mathrm{r} 1 \neq \mathrm{r} 2 \neq \mathrm{r} 3$, which will randomly generate for each variation vector. Scale factor $\mathrm{F}$ is a positive real constant, it controls the difference vector scaling, $\mathrm{F} \in$ $[0,2]$.

(3) Crossover operator. The crossover operation is to generate a new test vector $\mathrm{U}_{\mathrm{iG}}=\left(\mathrm{U}_{1 \mathrm{i}, \mathrm{G}}, \mathrm{U}_{1 \mathrm{i}, \mathrm{G}}, \ldots, \mathrm{U}_{\mathrm{Di}, \mathrm{G}}\right)$ by mixing each dimension parameter of mutation vector $\mathrm{V}_{\mathrm{ig}}$ and objective vector $\mathrm{X}_{\mathrm{iG}}$ according to certain rules. In the $\mathrm{DE}$ algorithm, binomial cross is often used, the mathematical expression is:

$$
U_{j i, G}=\left\{\begin{array}{ll}
V_{j i, G}, & \text { if } \text { rand }_{j}(\mathrm{O}, 1) \leq C R \text { or } j=k \\
X_{j i, G}, & \text { otherwise }
\end{array}, j=1,2, \cdots, D\right.
$$

Where, $C R \in[0,1]$ is a real crossover probability constant value provided by the user, same with difference zoom factor F, which is a DE algorithm of control parameter; $k \in[1, D]$ and randomly selected to ensure that at least one dimension of the test vector $U_{i \mathrm{i}}$ is contributed by variation vector $\mathrm{V}_{\mathrm{iG}}$.

(4) Selection operator. After crossover and mutation, test vector $\mathrm{U}_{\mathrm{iG}}$ are competing with the target vector $X_{i G}$ to select the individual as the offspring of the individual with better fitness value. Assuming the objective function is minimized, mathematical representation of its selection procedures are as follows:

$$
X_{i, G+1}= \begin{cases}U_{i, G}, & \text { if } f\left(U_{i, G}\right) \leq f\left(X_{i, G}\right) \\ X_{i, G}, & \text { otherwise }\end{cases}
$$

Where $\mathrm{f}(\cdot)$ represents the fitness function.

DE algorithm is mainly involved in population size NP, differential scale factor $\mathrm{F}$ and crossover probability CR three control parameters. Selecting the appropriate control parameters is critical to the performance of the algorithm. So far the research show that population size $\mathrm{NP} \in[4 \mathrm{D}, 10 \mathrm{D}]$ is more appropriate, scale factor and crossover probability in the range $\mathrm{F} \in[0.4,1]$ and $\mathrm{CR} \in[0.5,0.95]$ are more effective.

\subsection{DE Algorithm Improvements}

DE algorithm is a simple and strong searching evolutionary algorithm. To standard test functions and practical engineering problems, DE algorithm with fast convergence speed and high quality solution is better than the other evolutionary algorithms. Although compared to other evolutionary algorithms, DE has a relatively good performance of the algorithm, but for complex engineering optimization problems, DE algorithm show the solution quality is not high or slow convergence problem. The reason for this result is the DE algorithm cannot effectively use objective function information that has been obtained.

For WSN distribution optimization problem, this paper proposes a hybrid differential evolution algorithm based on quadratic interpolation. The algorithm is embedded simplifying quadratic interpolation (SQI) method to improve the overall performance of original DE algorithm in the framework of the DE algorithm. This method is designed to improve the local search capabilities, and reduce the computational cost.

Simplifying quadratic interpolation method is a simple and effective search method, it is not need derivative information of target function, computing simple and suitable as a heuristic search operator. In this paper, as a local search operator it is embedded in the DE algorithm to accelerate standard DE convergence and improve the accuracy of the solution. SQI mathematical expressions is as follows: 


$$
p_{\cdot j}=\frac{1}{2}\left[\frac{\left(x_{2 i}^{2}-x_{3 i}^{2}\right) f\left(x_{1}\right)+\left(x_{3 i}^{2}-x_{1 i}^{2}\right) f\left(\mathrm{x}_{2}\right)+\left(x_{1 i}^{2}-x_{2 i}^{2}\right) f\left(x_{3}\right)}{\left(x_{2 i}-x_{3 i}\right) f\left(x_{1}\right)+\left(x_{3 i}-x_{1 i}\right) f\left(\mathrm{x}_{2}\right)+\left(x_{1 i}-x_{2 i}\right) f\left(x_{3}\right)}\right], \quad i=1,2, \cdots D
$$

Where $x_{j}=\left(x_{j 1}, x_{j 2}, \cdots x_{j D}\right)^{T}, \mathrm{j}=1,2,3, \ldots . \mathrm{D}$ is the D-dimensional test point; $p=\left(p_{\cdot 1}, p_{\cdot 2}, \cdots, p_{\cdot D}\right)^{T}$ is new test point calculated by formula $(13) ; \mathrm{f}(\cdot)$ is the objective function to solve problem. Pseudo code of SQI local search operators is shown in the following table 1 . Setting LocalK less than or equal to MaxN is to avoid the program in an endless loop, MaxN represents the maximum number of SQI calculation. From the experimental results, when the population size of the evolutionary algorithms (NP) is less than 30, it is recommended to take MaxN 1; Otherwise, MaxN take 3.

\section{Table 1. Pseudo Code of SQI Local Search Operators}

Step1 find the best, worst point and their fitness values in the NP points: $b, w$, $\mathrm{f}_{\mathrm{b}}, \mathrm{f}_{\mathrm{w}}($ The best, worst, best and worst fitness). Set LocalK $=1$.

Step2 DO $\mathrm{x}_{1}=\mathrm{b}$.

Step2.1 randomly selected from the NP points $\mathrm{x}_{2}$ and $\mathrm{x}_{3}$, and $\mathrm{x}_{2} \neq \mathrm{x}_{3} \neq \mathrm{b}$,

Step2.2 According to equation (13) to calculate the new test point $p=\left(p_{\cdot 1}, p_{\cdot 2}, \cdots, p_{\cdot D}\right)^{T}$.

Step2.3 calculate the value of $f_{P}$ and sets LocalK=LocalK +1 .

WHILE $\left(\mathrm{f}_{\mathrm{P}}<\mathrm{f}_{\mathrm{W}}\right)$, or LocalK $\left.\leq \operatorname{MaxN}\right)$

Step3 if $\mathrm{f}_{\mathrm{P}}<\mathrm{f}_{\mathrm{W}}$, then replace the point $\mathrm{w}$ with point $\mathrm{p}$.

\section{Hybrid Differential Evolution Improved Algorithm Steps Based on Quadratic Interpolation}

In summary, for the WSN network distribution optimization, the paper presents a hybrid differential evolution improved algorithm based on quadratic interpolation. The method implementation process is as follows:

1th step Parameter settings: population size NP, scale factor f, crossover probability $\mathrm{CR}$, maximum number of evolutionary Gmax.

2nd step Initial population which population size is NP is randomly generated, and the fitness value of NP individual is obtained.

3rd step Determine whether termination criterion is met, if satisfied, the algorithm terminates, go to the 6th step, otherwise turning to the 4th step.

4th step According to the formula, DE mutation, crossover and selection operation are performed on the current population of individuals.

5th step Perform local searching algorithms SQI.

6th step Output obtained the best results.

\section{Simulation and Analysis}

\subsection{Parameters Setting}

In this paper, the simulation experiment is carried out by using MATLAB 7 environment, the number of individual population NP is 40 , the maximum evolution 
algebra is 150 , the scaling factor $\mathrm{F}=0.5$ and the crossover probability $\mathrm{CR}=0.9$. The effective sensing radius of the sensor nodes is $12 \mathrm{~m}$, and the optimization of the distribution of the 30 mobile sensor nodes in the monitoring area of $100 * 100$ is simulated. The simulation results of two optimization algorithms are shown in Figure 2.

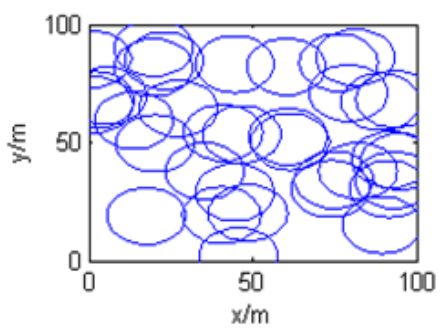

(a)Initial node distribution

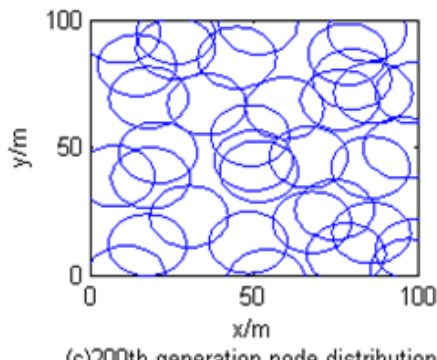

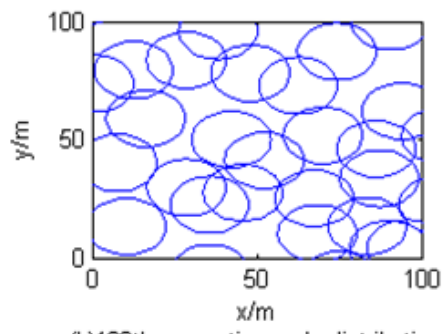

(b)100th generation node distribution

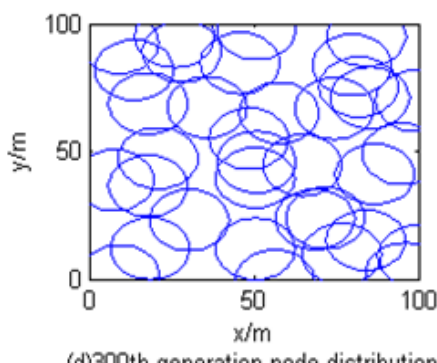

(d)300th generation node distribution

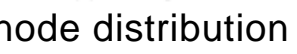

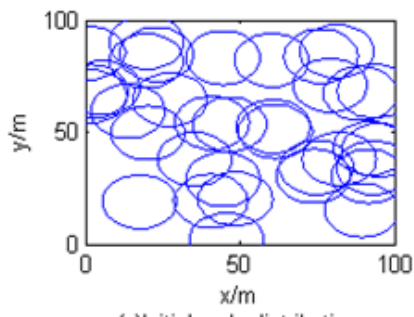

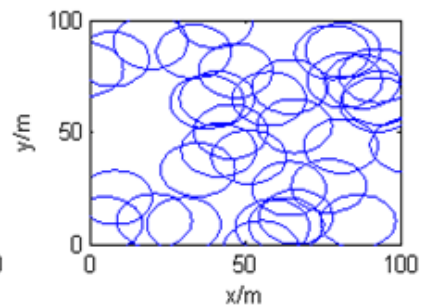

(a)Initial node distribution

(b)100th generation node distribution

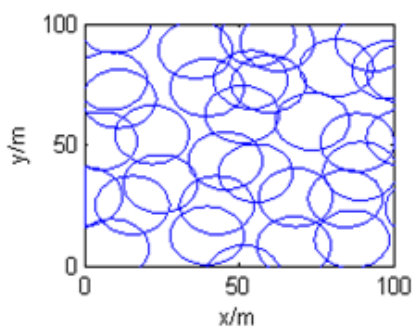

(c)200th generation node distribution

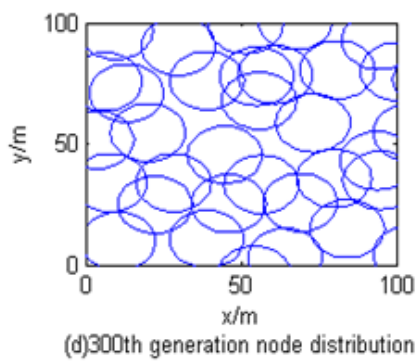

(b) improved DE algorithm node distribution

\section{Figure 2. DE and Improved DE Algorithm Node Optimization Distribution}

\subsection{Simulation Analysis}

Under the different sensor node density and different iterations, to observe coverage rate of WSN nodes based on DE optimization algorithm and improved algorithm of this paper, we can see from the Figure 3 that, in the case of the same coverage rate, DE improved algorithm requires fewer nodes than the standard DE algorithm, when the coverage rate needs to reach 95\%, the required nodes of DE improved algorithm is 10 less than the standard DE algorithm. Figure 4 show that under the same conditions, the DE improved algorithm tends to converge after 200 times of iteration, the standard DE algorithm tends to converge after 400 times of iteration, the DE improved distribution 
optimization algorithm converges faster than standard DE algorithm, and the coverage rate also higher than original DE algorithm.

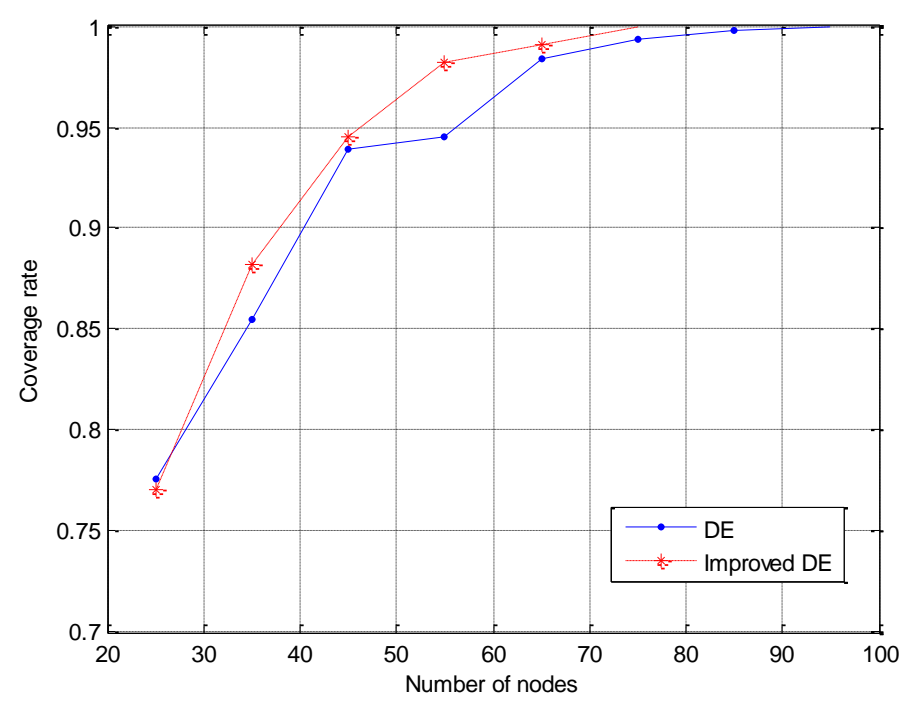

Figure 3. Coverage Rate Comparison of Different Number Of Nodes

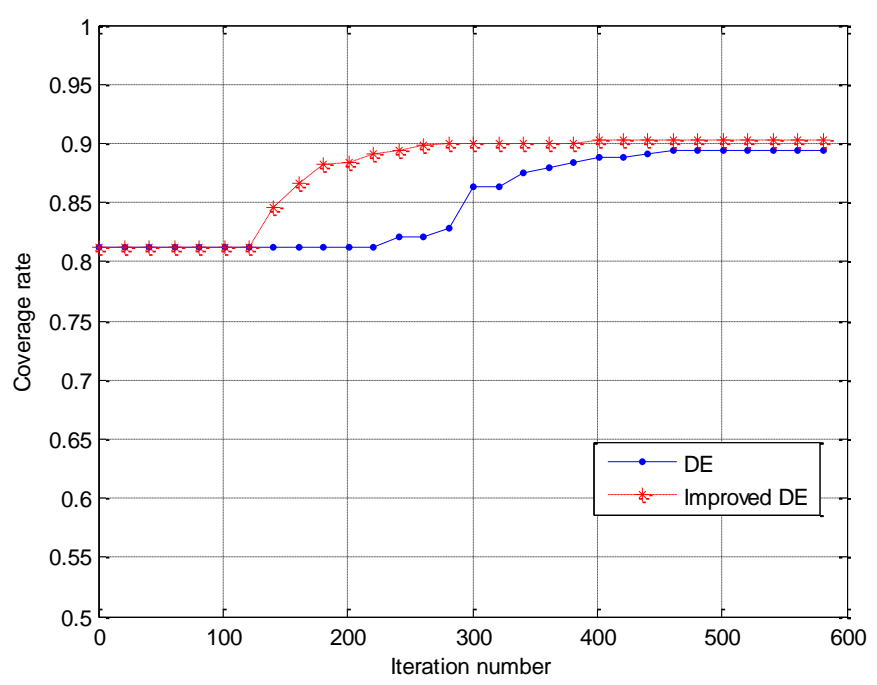

Figure 4. Coverage Rate Comparison of Different Iteration Number

\section{Conclusion}

Network optimization is an important issue in wireless sensor networks, and it is the basis of further research on the wireless sensor network. WSN coverage optimization, which can more reasonable allocate network resources, and has a vital role on environmental awareness and information accessing. This paper presents an improved wireless sensor networks effective coverage method based on differential evolution algorithm, which using quadratic interpolation method to improve the algorithm performance to further optimize the effective coverage of wireless sensor network nodes. Simulation results show that, compared to DE algorithm, the new algorithm can improve local search capabilities, reduce the computational cost of the algorithm, accelerate 
standards DE convergence, improve the accuracy of solution, and has some reference value for optimization application of wireless sensor network.

\section{Acknowledgments}

The authors would like to thank all the reviewers for their constructive comments. The authors acknowledge with thanks the Scientific Research Program Funded by Shaanxi Provincial Education Department (Program No. 14JK1829), Yan'an Municipal Science and Technology Research and Development Program (Program No. 2014KG-04), the Science and Technology Research and Development Program Project in Shaanxi Province (Industrial Research) (Grant No.2016GY-138), and the National Nature Science Foundation of China (Grant No. 61379026).

\section{References}

[1] A. Howard, M. J. Mataric and G. S. Sukhamae, "An incremental self-deployment algorithm for mobile sensor networks", Autonomous Robots, vol. 13, no. 2, (2002), pp. 113-126.

[2] N. Heo and P. K. Varshney, "Energy-efficient deployment of intelligent mobile sensor networks", IEEE Transactions on Systems, Man, and Cybernetics Part A: Systems and Humans, vol. 35, no. 1, (2005), pp. 78-92.

[3] R. Mallipeddi R, Suganthan P N. Empirical study on the effect of population size on differential evolution algorithm", CEC 2008. (IEEE World Congress on Computational Intelligence), IEEE Congress on Evolutionary Computation, IEEE, (2008), pp. 3663-3670.

[4] T. V. Neri, "Recent advances in differential evolution: a survey and experimental analysis", Artificial Intelligence Review, vol. 33, no. 1-2, (2010), pp. 61-106.

[5] Z. Q. Pei, C. Q. Xu and J. Teng, "Relocation algorithm for nonuniform distribution in mobile sensor network", Journal of Electronics, vol. 26, no. 2, (2009), pp. 222-228.

[6] X. J. Xu, X. P. Huang and D. L. Qian, "Adaptive accelerating differential evolution", Complex Systems and Complexity Science, vol. 5, no. 1, (2008), pp. 87-92.

[7] K. Chakrabarty, S. S. Iyengar and H. R. Qi, "Grid coverage for surveillance and target location in distributed sensor networks", IEEE Transactions on Computers, vol. 51, no. 12, (2002), pp. 1448-1453.

[8] X. F. Li, Y. C. Mao, Y. Liang, "A survey on topology control in wireless sensor networks", In: Proceedings of the 10th International Conference on Control, Allocation, Robotics and Vision, ICARCV 2008, Hanoi, Viet, (2008), pp. 251-255.

[9] C. Karlof and D. Wagner, "Secure routing in wireless sensor networks: attacks and countermeasures", Ad Hoc Networks, vol. 1, no. 1, (2003), pp. 293-315.

[10] M. Mostafizur, R. Mozumdar and G. F. Nan, "An efficient data aggregation algorithm for cluster-based sensor network", Journal of Networks, vol. 4, no. 7, (2009), pp. 598-606.

[11] N. H. Malka, M. G. Siddeswara and J. Andrew, "Centralised strategies for cluster formation in sensor networks", Studies in Computational Intelligence, vol. 4, (2005), pp. 315-331

[12] M. Cardei and J. Wu, "Coverage in wireless sensor networks", Handbook of Sensor Networks, CRC Press, (2004).

\section{Authors}
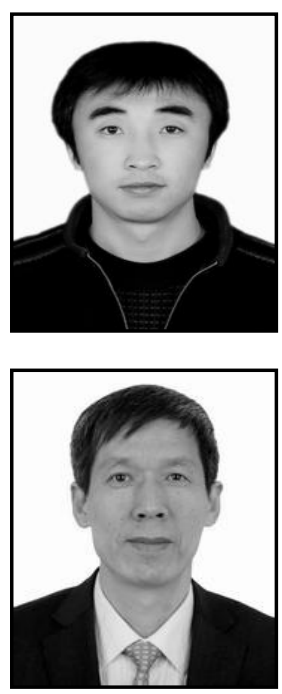

Wenli Lei, he received the M.S degree from Xi an University of Science and Technology in 2008. His research interests include wireless sensor network, artificial intelligence, and so on.

Fubao Wang, his research interests include computer networks, network and information security, wireless communication networks, digital multimedia communications and so on. 
International Journal of Future Generation Communication and Networking Vol. 9, No. 11 (2016) 